\title{
Monomial tropical cones for multicriteria optimization
}

Cite as: AIP Conference Proceedings 2070, 020025 (2019); https://doi.org/10.1063/1.5089992

Published Online: 12 February 2019

Michael Joswig, and Georg Loho

ARTICLES YOU MAY BE INTERESTED IN

Lower and upper bounds for the general multiobjective optimization problem

AIP Conference Proceedings 2070, 020038 (2019); https://doi.org/10.1063/1.5090005

Multi-objective mixed integer programming: An objective space algorithm

AIP Conference Proceedings 2070, 020039 (2019); https://doi.org/10.1063/1.5090006

Using a B\&B algorithm from multiobjective optimization to solve constrained optimization problems

AIP Conference Proceedings 2070, 020028 (2019); https://doi.org/10.1063/1.5089995

\section{Lock-in Amplifiers up to $600 \mathrm{MHz}$

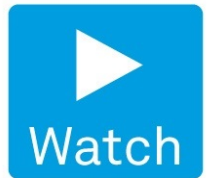

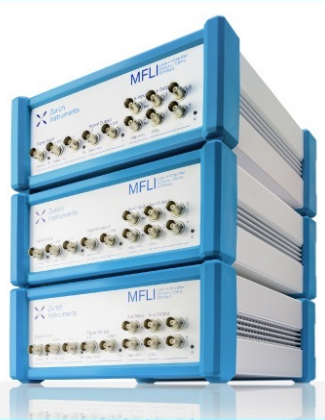




\title{
Monomial Tropical Cones for Multicriteria Optimization
}

\author{
Michael Joswig ${ }^{1, b), *}$ and Georg Loho $^{2, a), \dagger}$ \\ ${ }^{1}$ Institut für Mathematik, MA 6-2, TU Berlin, Str. des 17. Juni 136, 10623 Berlin, Germany \\ ${ }^{2}$ EPFL SB MATHAA DISOPT, MA C1 563 (Bâtiment MA), Station 8, CH-1015 Lausanne \\ ${ }^{a)}$ Corresponding author: georg.loho@epfl.ch \\ b) joswig@math.tu-berlin.de
}

\begin{abstract}
We present an algorithm to compute all $n$ nondominated points of a multicriteria discrete optimization problem with $d$ objectives using at most $O\left(n^{\lfloor d / 2\rfloor}\right)$ scalarizations. The method is similar to an algorithm by Klamroth et al. (2015) with the same complexity. As a difference, our method employs a tropical convex hull computation, and it exploits a particular kind of duality which is special for the tropical cones arising.
\end{abstract}

\section{INTRODUCTION}

The general form of a multicriteria optimization problem reads

$$
\begin{array}{ll}
\min & f(x)=\left(f_{1}(x), \ldots, f_{d}(x)\right) \\
\text { subject to } & x \in X .
\end{array}
$$

Here $X$ is the feasible set. It is a subset of the decision space, which may be any set. The objective functions $f_{i}$ have the feasible set as their common domain, and they take real values. We will mainly deal with the outcome space $Z=f(X)$, which is a subset of $\mathbb{R}^{d}$. A point $z \in Z$ is nondominated if there is no point $w \in Z$ such that $w_{i} \leq z_{i}$ for all $i \in[d]$ and $w_{\ell}<z_{\ell}$ for at least one $\ell \in[d]$. The set of all nondominated points in $Z$ is the nondominated set. Our goal is to describe an algorithm for finding the nondominated set of an arbitrary discrete multicriteria optimization problem.

Tropical geometry is a recent branch of mathematics in the middle of algebraic geometry, combinatorics and optimization [1]. This connection is particularly fruitful for optimization. First, many classical topics in optimization and complexity theory receive a geometric interpretation, and this leads to a deeper understanding. For instance, the decision problem MEAN-PAYOFF is equivalent to deciding the feasibility of a tropical linear program [2]; this is interesting as MEAN-PAYOFF lies in the complexity class NP $\cap$ co-NP, but no polynomial time algorithm is known. Second, techniques from optimization can be applied to solve problems in geometry. For instance, the Hungarian method provides an efficient method for computing tropical determinants [3, \$1.6.4]. Here we add a new page on multicriteria optimization to the dictionary which translates between tropical geometry and optimization.

Each nondominated point can be obtained by determining an optimal solution of a scalarization of the multiobjective problem [4]. There are general methods known to determine all nondominated points by successively choosing appropriate scalarizations. These scalarizations are considered computationally expensive, whence the complexity of a multicriteria optimization problem is measured in the number of scalarizations required. Our algorithm can be viewed as a variation of a method by Klamroth et al. [5] of optimal complexity $O\left(n^{\lfloor d / 2\rfloor}\right)$ for $d$ fixed [6]. The new contribution is the observation that the nondominated set can be interpreted as the extremal generators of a certain kind of tropical cone. Adaptating the tropical double description method [7] allows us to derive an enumeration scheme, which is asymptotically worst case optimal. In this way the upper bound [6] becomes a consequence of McMullen's upper bound theorem [8]; see also [9]. The full version of the paper can be found at [10]; it is submitted for publication.

\footnotetext{
${ }^{*}$ Research by M. Joswig is carried out in the framework of Matheon supported by Einstein Foundation Berlin. Further support by DFG (SFB-TRR 109: "Discretization in Geometry and Dynamics" and SFB-TRR195: "Symbolic Tools in Mathematics and their Application")

${ }^{\dagger}$ G. Loho was partially supported by the Swiss National Science Foundation (SNSF) within the project "Convexity, geometry of numbers, and the complexity of integer programming" (Nr. 163071).
} 


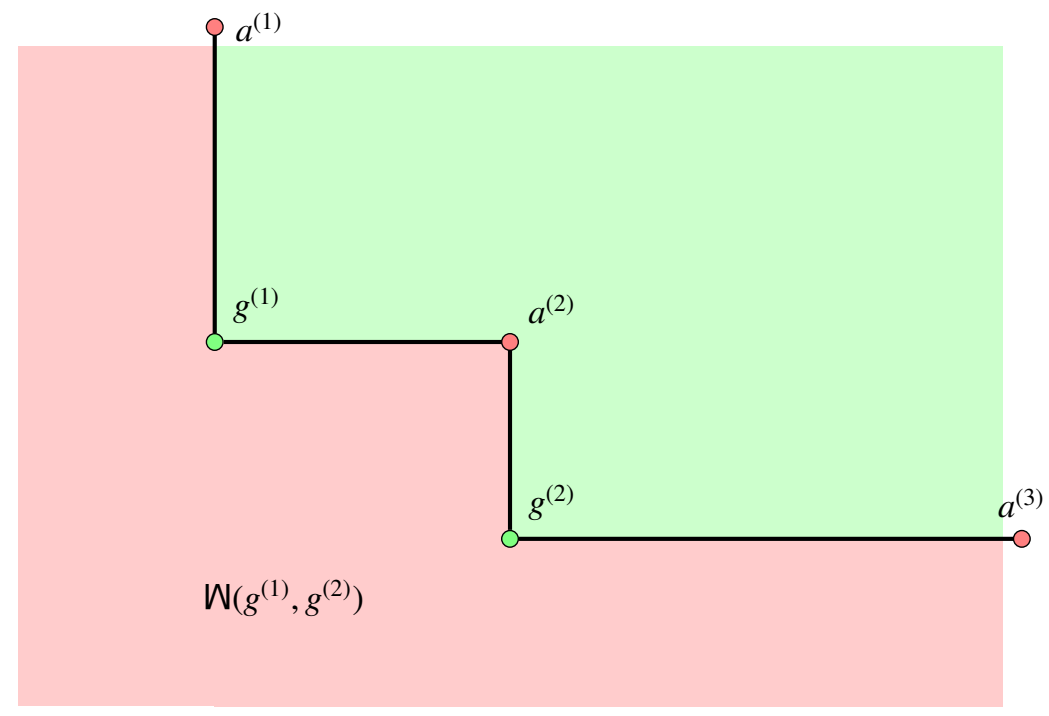

FIGURE 1: The plane is separated by a 'crooked line' in two parts. Each part is the union of non-negative or nonpositive orthants shifted by $g^{(1)}, g^{(2)}$ and $a^{(1)}, a^{(2)}, a^{(3)}$, respectively. The apices $a^{(1)}$ and $a^{(3)}$ lie at infinity.

\section{A COMPLEMENTARY PAIR OF TROPICAL CONES}

Our algorithm for multicriteria optimization is fundamentally based on a geometric observation, which we explain now. The tropical semiring $\mathbb{T}_{\min }$ is the set $\mathbb{R} \cup\{\infty\}$ equipped with min as the tropical addition and the standard addition as the tropical multiplication. There is a dual version, $\mathbb{T}_{\max }$, where max is the addition, and $-\infty$ is the neutral element with respect to max. Note that $x \in \mathbb{T}_{\min }$ if and only if $-x \in \mathbb{T}_{\max }$. Throughout the following we fix an integer $d \geq 1$. A min-tropical cone $C$ is a nonempty subset of $\mathbb{T}_{\min }^{d+1}$ which is closed with respect to taking min-tropical scalar combinations, i.e.,

$$
\left(\min \left(\lambda+x_{0}, \mu+y_{0}\right), \ldots, \min \left(\lambda+x_{d}, \mu+y_{d}\right)\right) \in C \quad \text { for all } \lambda, \mu \in \mathbb{T}_{\min } \text { and } x, y \in C .
$$

It follows that any min-tropical cone contains the point $(\infty, \infty, \ldots, \infty)$. Notice that we take indices $0,1, \ldots, d$ for vectors in $\mathbb{T}_{\min }^{d+1}$. A set $G \subset \mathbb{T}_{\min }^{d+1}$ is said to generate the min-tropical cone $C$ if this is the smallest min-tropical cone which contains $G$. Scaling the generators tropically, i.e., adding multiples of the all-ones-vector $\mathbf{1}$ does not change the tropical cone. If $C$ is finitely generated, then there is a generating set which is minimal with respect to inclusion; and this is unique, up to tropical scaling; cf. [3, Prop. 3.3.6]. The elements of that minimally generating set are the extremal generators of $C$. The same notions also exist with max instead of min. Let $\mathbb{R}_{\geq 0}^{d}$ and $\mathbb{R}_{>0}^{d}$ denote the $d$-dimensional nonnegative and positive orthant, respectively.

Theorem 1 ([10, Theorem 10]) Let $G$ be a finite subset of $\mathbb{R}^{d}$. Then there is a finite set $A \subseteq \mathbb{T}_{\min }^{d}=(\mathbb{R} \cup\{\infty\})^{d}$ such that

$$
\mathrm{M}(G):=\bigcup_{g \in G} g+\mathbb{R}_{\geq 0}^{d}=\mathbb{R}^{d} \backslash\left(\bigcup_{a \in A}\left(a-\mathbb{R}_{>0}^{d}\right)\right)
$$

Moreover, $A$ is the set of finite extremal generators of the min-tropical cone

$$
\mathrm{W}(G):=\bigcap_{g \in G}\left(\mathbb{R}^{d} \backslash\left(g+\mathbb{R}_{>0}^{d}\right)\right) .
$$

The representation described in Theorem 1 is visualized in Figure 1. The closure of each part is tropically convex, but in two distinct ways; the lower part $\mathrm{W}(G)$ is min-tropically convex, while the upper part $\mathrm{M}(G)$ is max-tropically convex. We call them monomial tropical cones. 


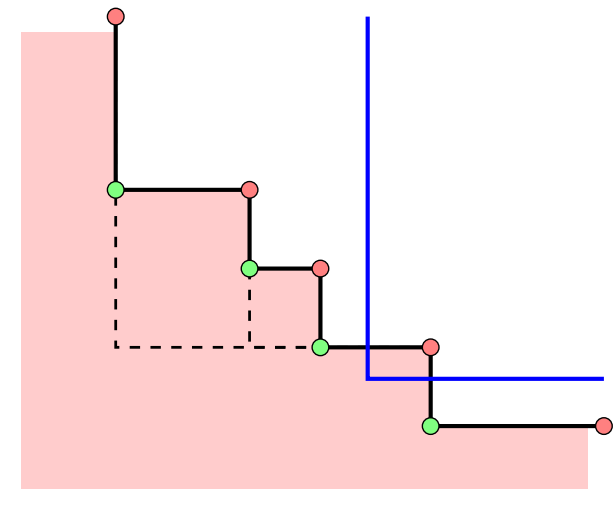

(a) Monomial tropical cone with new trop. halfspace

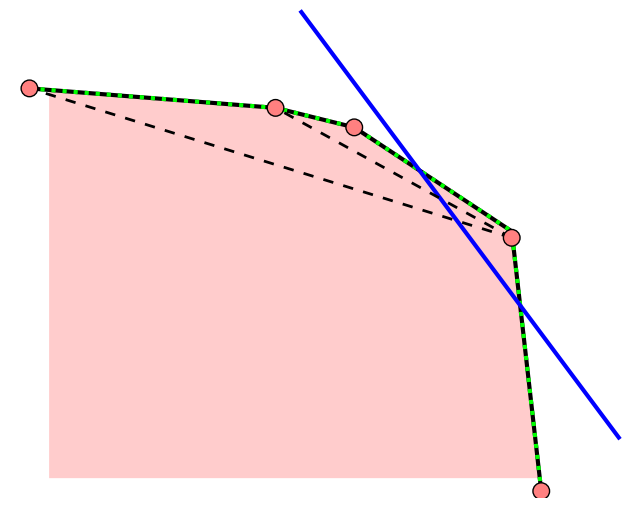

(c) Unbounded polygon with new halfspace

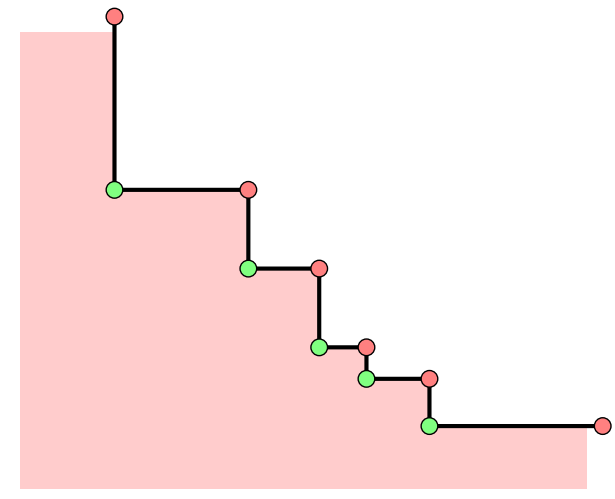

(b) Updated monomial tropical cone

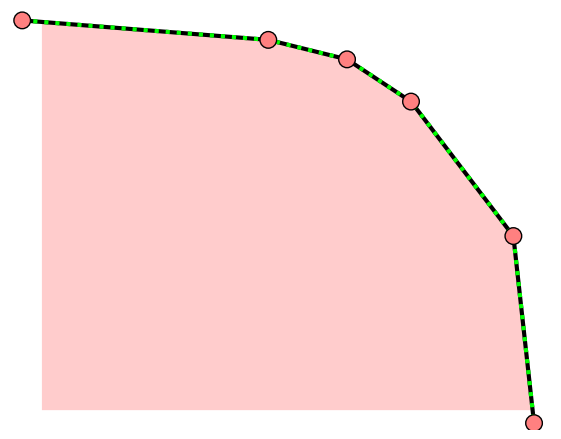

(d) Updated unbounded polygon

FIGURE 2: In $2 \mathrm{a}$ and $2 \mathrm{~b}$, we sketch one iteration in a dual tropical convex hull computation as it arises in our algorithm. The blue orthant in 2 a corresponds to a new nondominated point found which gives rise to an additional min-tropical linear inequality. The result is shown in $2 \mathrm{~b}$. The bottom row ( $2 \mathrm{c}$ and $2 \mathrm{~d})$ sketches an analogous situation in the ordinary setting (without being metrically correct). The new extremal generators in $2 \mathrm{~b}$ and $2 \mathrm{~d}$ arise as intersections of the blue (tropical) halfspace with the dashed lines in $2 \mathrm{a}$ and $2 \mathrm{c}$. These connect remaining extremal generators and points which are cut off.

\section{GENERATING ALL NONDOMINATED POINTS}

We now address the multicriteria optimization problem (1), and we assume that it is discrete, i.e., $Z=f(X) \subset \mathbb{R}^{d}$ is finite. It is common to rely on scalarization oracles which either produce a new nondominated point or certify that a certain portion of the search region does not contain any. More specifically, as in [5, Algorithm 1], we can employ scalarizations like the ' $\epsilon$-constraint method' [4, $\$ 4.1]$.

Proposition 2 The nondominated set $N$ agrees with the set of those extremal generators of the max-tropical cone $\mathrm{M}(N)=\mathrm{M}(Z)$ which have finite coordinates. Moreover, if $A$ is the set of extremal generators of the complementary min-tropical cone $\mathrm{W}(N)$, then the set $\bigcup_{a \in A} a-\mathbb{R}_{>0}^{d}$ agrees with the complement of $\mathrm{M}(N)$ in $\mathbb{R}^{d}$.

It turns out that there are only $d$ minimal generators of $\mathrm{M}(Z)$ which have infinite coordinates, and these are known a priori. Therefore, to determine all minimal generators of that max-tropical cone is exactly the same as finding all nondominated points.

Now our algorithm is the following. Throughout we maintain a pair of sets, $G$ and $A$, which describe a complementary pair of tropical cones as in Theorem 1. From $A$ we iteratively generate scalarizations, which may or may not yield a new nondominated point, $g$. If we obtain a new $g$, then it is added to $G$; afterwards we update the outer 
description of the max-tropical cone $\mathrm{M}(G)$, which is the same as a generating set of the min-tropical cone $\mathrm{W}(G)$. The latter generating set becomes the new $A$. The algorithm terminates once we know that $\bigcup_{a \in A} a-\mathbb{R}_{>0}^{d}$ does not contain any nondominated point. We finally return the current set $G$, and this is now $N$, the nondominated set of (1).

Since the minimal generators of $\mathrm{M}(N)$ correspond to a minimal description of $\mathrm{W}(N)$ in terms of tropical linear inequalities the above method amounts to iteratively solving a tropical convex hull problem for $\mathrm{M}(N)=\mathrm{M}(Z)$. Equivalently, this amounts to solving the dual tropical convex hull problem for $W(N)$. One standard algorithm for computing (dual) tropical convex hulls is the tropical double description method in [7], depicted in Figure 2. This is a direct analog of the double description method, also known as Fourier-Motzkin elimination for ordinary polyhedral cones; cf. [11]. Thanks to the correspondence between ordinary and tropical cones via fields of real Puiseux series (cf. [12] and [13]) essentially the same complexity bounds apply in the tropical and in the ordinary setting. In this way, McMullen's classical upper bound theorem [8] implies that the size of the set $A$ in our algorithm is at most of order $O\left(n^{\lfloor d / 2\rfloor}\right)$, where $n=\# N$ is the number of nondominated points and $d$ is considered a fixed constant; see also [9]. Since each scalarization enlarges $G$ or certifies, that the portion of the search area corresponding to a point in $A$ does not contain a nondominated point, we arrive at the following complexity bound, which is the same as in Klamroth et al. [5, 14]; work of Kaplan et al. [6] implies that the bound is worst-case optimal.

Theorem 3 For fixed d, the maximal number of scalarizations required to enumerate all nondominated points lies in $\Theta\left(n^{\lfloor d / 2\rfloor}\right)$.

\section{CONCLUSION}

Interpreting discrete multicriteria optimization in terms of tropical convexity leads to a natural algorithm for finding the nondominated set, and its complexity, measured by the number of scalarizations required, is worst-case optimal. It also reveals a new relationship between multicriteria optimization and the computation of convex hulls. In view of [15] this looks like a promising direction for future research.

\section{ACKNOWLEDGMENTS}

We would like to thank Ben Burton and Kathrin Klamroth for helpful comments.

\section{REFERENCES}

[1] D. Maclagan and B. Sturmfels, Introduction to tropical geometry, Graduate Studies in Mathematics, Vol. 161 (American Mathematical Society, Providence, RI, 2015), pp. xii+363.

[2] M. Akian, S. Gaubert, and A. Guterman, Internat. J. Algebra Comput. 22, 1250001, 43 (2012).

[3] P. Butkovič, Max-linear systems: theory and algorithms, Springer Monographs in Mathematics (SpringerVerlag London, Ltd., London, 2010), pp. xviii+272.

[4] M. Ehrgott, Multicriteria optimization, 2nd ed. (Springer-Verlag, Berlin, 2005), pp. xiv+323.

[5] K. Klamroth, R. Lacour, and D. Vanderpooten, European J. Oper. Res. 245, 767-778 (2015).

[6] H. Kaplan, N. Rubin, M. Sharir, and E. Verbin, SIAM J. Comput. 38, 982-1011 (2008).

[7] X. Allamigeon, S. Gaubert, and E. Goubault, in STACS 2010: 27th International Symposium on Theoretical Aspects of Computer Science, LIPIcs. Leibniz Int. Proc. Inform., Vol. 5 (Schloss Dagstuhl. Leibniz-Zent. Inform., Wadern, 2010), pp. 47-58.

[8] P. McMullen, Mathematika 17, 179-184 (1970).

[9] X. Allamigeon, S. Gaubert, and R. D. Katz, J. Combin. Theory Ser. A 118, 162-189 (2011).

[10] M. Joswig and G. Loho, Monomial tropical cones for multicriteria optimization, (2017), 1707.09305 .

[11] K. Fukuda and A. Prodon, in Combinatorics and computer science (Brest, 1995), Lecture Notes in Comput. Sci., Vol. 1120 (Springer, Berlin, 1996), pp. 91-111.

[12] M. Develin and J. Yu, Experiment. Math. 16, 277-291 (2007).

[13] M. Joswig, G. Loho, B. Lorenz, and B. Schröter, in Mathematical aspects of computer and information sciences, Lecture Notes in Comput. Sci., Vol. 9582 (Springer, [Cham], 2016), pp. 429-445.

[14] K. Dächert, K. Klamroth, R. Lacour, and D. Vanderpooten, European J. Oper. Res. 260, 841-855 (2017).

[15] A. Löhne and B. Weißing, Math. Methods Oper. Res. 84, 411-426 (2016). 